\title{
DEPTH-INTEGRATED NUMERICAL MODELING OF TURBULENT TRANSPORT BY LONG WAVES AND CURRENTS
}

\begin{abstract}
Dae-Hong Kim $^{1}$ and Patrick Lynett ${ }^{2}$
In nature, flows are 3D phenomenon, but, in many geophysical settings, the water depth scale is smaller relative to the horizontal scale, such that horizontal 2D (H2D) motions dominate the flow structure. In those cases, especially in large domains, the H2D numerical model can be a practical and accurate tool - if the 3D physical properties can be included properly into the H2D model. Some of the H2D approaches in widespread use are the Boussinesq-type equations (BE) and shallow water equations (SWE) derived by a perturbation approach or depth averaging. The BE can account for some of the dispersive, turbulent and rotational flow properties frequently observed in nature (Kim et al., 2009). Also it has the ability of coupling currents and waves and can predict nonlinear water wave propagation over an uneven bottom from deep (or intermediate) water to the shallow water area. However, during the derivation of a H2D equation set, BE or SWE, some of the 3D flow properties like the dispersive stresses (Kuipers and Vreugdenhill, 1973) and the effects of the unresolved small scale 3D turbulence are excluded. Subsequently, there must be some limitations for predicting horizontal flow structures which can be generated through these neglected 3D effects. Naturally, any inaccuracy of the hydrodynamic flow model is reflected in the results of a coupled scalar transport model. In order to incorporate 3D turbulence effects into H2D flow models, various approaches have been proposed. Among many others, the stochastic backscatter model (BSM) proposed by Hinterberger et al. (2007) can account for the mechanism of inverse energy transfer from unresolved 3D turbulence to resolved 2D flow motions. Reasonable results were obtained by the proposed methods. Similar to the flow model, for scalar transport it is desired to develop a H2D model that can approximately account for the vertical deviations of concentration and velocity, and the associated mixing. For the accurate prediction of transport, an accurate numerical solver which can minimize numerical dispersion, dissipation and diffusion should be developed. Recently, the finite volume method (FVM) using approximate Riemann solvers has been developed and applied successfully. In this study, a depth-integrated model including subgrid scale mixing effects for turbulent transport by long waves and currents is presented. A fullynonlinear, depth-integrated set of equations for weakly dispersive and rotational flow are derived by the long wave perturbation approach. The same approach is applied to derive a depth-integrated scalar transport model. The proposed equations are solved by a fourth-order accurate FVM. The depth-integrated flow and transport models are applied to typical problems which have different mixing mechanisms. Several important conclusions are obtained from the simulations: (i) From a mixing layer simulation it is revealed that the dispersive stress implemented with a stochastic BSM plays an important role for energy transfer. (ii) The proposed transport model coupled with the depthintegrated flow model can predict the passive scalar transport based on the turbulent intensity - not by relying on empirical constants. (iii) For near field transport simulations, the inherent limitation of the two-dimensional horizontal model to capture vertical structure is recognized. (iv) If the main mechanism of flow instability originates from relatively large-scale bottom topography features, then the effects of the dispersive stresses are less important.
\end{abstract}

Keywords: turbulent transport, Boussinesq-type equations

\section{INTRODUCTION}

In nature, flows are 3D phenomenon. However, in many cases of the geophysical flows, the water depth is limited relative to the horizontal scale so that the horizontal 2D motions dominate the flow structures. In those cases, especially in large domains, the horizontal 2D numerical model can be a practical and accurate tool if the 3D physical properties can be reflected properly into the 2D model. One of the 2D approaches mostly wide spread are the Boussinesq equations and shallow water equations with the long wave scaling derived by perturbation approach or depth averaging.

The Boussinesq equations model can account the dispersive, turbulent and rotational flow properties frequently observed in nature (Kim et al., 2009). Also it has the ability of coupling the currents and waves (Yoon and Liu, 1989) and can predict the nonlinear wave propagations over uneven bottom from deep (or intermediate) water area to shallow water area (Nwogu, 1993 and Wei et al. 1995).

However, during the derivations of a 2D horizontal equation set, some 3D flow features such as the dispersive stresses (Kuipers and Vreugdenhill, 1973) and the effects of the unresolved small scale 3D turbulence are excluded. Consequently, there must be some limitations for predicting the horizontal flow structures which can be originated by the neglected 3D effects. Naturally, the inaccuracy of the flow model is reflected in the results of a transport model.

\footnotetext{
${ }^{1}$ Department of Civil Engineering, University of Seoul, Seoul, Republic of Korea.

${ }^{2}$ Zachry Department of Civil Engineering, Texas A\&M University, College Station, Texas, USA
} 
In order to incorporate the 3D turbulence effects into 2D horizontal flow models, various approaches were proposed. For example, Nadaoka and Yagi (1998) incorporated a subdepth scale turbulence model based on an eddy viscosity into the shallow water equations. A stochastic BSM proposed by Hinterberger et al. (2007) can account the mechanism of the inverse energy transfer from the unresolved 3D turbulence to the resolved 2D flow motions. Reasonable results were obtained by the proposed methods.

Similar to the flow model, it is required to develop a 2D horizontal scalar transport model that can account the vertical deviations of the concentration and velocity. Taylor (1953) firstly proposed a brilliant method how to reflect the effects of the vertical nonuniformity into the 2D horizontal model. His result, commonly called 'dispersion', was extended to various environmental flow fields by many researchers (Fischer et al., 1979).

For the accurate prediction of transport, an accurate transport numerical solver which can minimize the numerical dispersion, dissipation and diffusion should be developed. Recently, the FVM using approximate Riemann solvers has been developed and applied successfully, for example, Mingham and Causon (2008). The FVM has many advantages. Especially, in the view of eigen structure, the advection equation has the same approximate Riemann solver with the equation of tangential velocity of homogeneous shallow water equations (Toro, 2002). Hence, the exactly same numerical method for the leading-order terms of the Boussinesq equations can be used for the advection terms of the transport equation with consistency.

In this paper, the turbulent transports by the long waves and currents is investigated. The Boussinesq-type equations with subgrid turbulence closure are introduced. In the next section, a depthintegrated transport equation is introduced. The numerical methods for the transport equation and the test results are briefly presented. The turbulent transport by a plane mixing layer and by bottom topography are presented in the following sections.

\section{DEPTH-INTEGRATED FLOW MODEL}

\section{Dispersive Stress by the velocity Fluctuation}

The 3D space, the spatially-filtered continuity and Navier-Stokes equations for incompressible flow are given by

$$
\begin{aligned}
& \frac{\partial \overline{\mathrm{u}}_{\mathrm{i}}^{*}}{\partial \mathrm{x}_{\mathrm{i}}^{*}}=0 \\
& \frac{\partial \overline{\mathrm{u}}_{\mathrm{i}}^{*}}{\partial \mathrm{t}^{*}}+\frac{\partial \overline{\mathrm{u}}_{\mathrm{i}}^{*} \overline{\mathrm{u}}_{\mathrm{j}}^{*}}{\partial \mathrm{x}_{\mathrm{j}}^{*}}+\frac{1}{\rho} \frac{\partial \overline{\mathrm{p}}^{*}}{\partial \mathrm{x}_{\mathrm{i}}^{*}}=\frac{\partial}{\partial \mathrm{x}_{\mathrm{i}}^{*}}\left(2 \nu \overline{\mathrm{S}_{\mathrm{lj}}^{*}}-\tau_{\mathrm{ij}}^{*}\right)
\end{aligned}
$$

where the overbar means the filtering operator. The subscripts $i, j=(1,2,3) . t$ is time and $u_{i}^{*}$ is the velocity vector, $\mathrm{p}^{*}$ is pressure, $\rho$ is the density of water, $v$ is the kinematic viscosity of water, $S_{\mathrm{ij}}^{*}$ is a strain rate tensor, and $\tau_{\mathrm{ij}}^{*}$ is the residual stress tensor. To derive the depth-integrated flow equations, a perturbation approach based on long wave scaling is used.

$$
\begin{aligned}
& (\mathrm{x}, \mathrm{y})=\frac{\left(\mathrm{x}^{*}, \mathrm{y}^{*}\right)}{\mathrm{l}_{\mathrm{o}}}, \quad \mathrm{z}=\frac{\mathrm{z}^{*}}{\mathrm{~h}_{\mathrm{o}}}, \quad \mathrm{t}=\frac{\mathrm{t}^{*} \sqrt{g \mathrm{~g}_{\mathrm{o}}}}{\mathrm{l}_{\mathrm{o}}}, \quad \mathrm{h}=\frac{\mathrm{h}^{*}}{\mathrm{~h}_{\mathrm{o}}}, \quad \zeta=\frac{\zeta^{*}}{\mathrm{~h}_{\mathrm{o}}}, \\
& (\mathrm{u}, \mathrm{v})=\frac{\left(\mathrm{u}^{*}, \mathrm{v}^{*}\right)}{\sqrt{\mathrm{gh}_{\mathrm{o}}}}, \quad \mathrm{w}=\frac{\mathrm{w}^{*}}{\mu \sqrt{\mathrm{gh}_{\mathrm{o}}}}, \quad \mathrm{p}=\frac{\mathrm{p}^{*}}{\rho g \mathrm{gh}_{\mathrm{o}}}, \quad \mu=\frac{\mathrm{h}_{\mathrm{o}}}{\mathrm{l}_{\mathrm{o}}}
\end{aligned}
$$

$\mathrm{h}^{*}$ is the water depth and $\zeta^{*}$ is the water surface elevation, which is a function of $\left(\mathrm{x}^{*}, \mathrm{y}^{*}, \mathrm{t}^{*}\right)$. $\mathrm{g}$ is the gravitational acceleration. A standard parameter for scale analysis of long waves is introduced and its magnitude is assumed to be $O\left(\mu^{2}\right) \ll 1.0$. The nondimensional horizontal eddy viscosity is given by

$$
v_{\mathrm{t}}^{\mathrm{h}}=\frac{v_{\mathrm{t}}^{\mathrm{h}^{*}}}{\alpha \mathrm{h}_{\mathrm{o}} \sqrt{\mathrm{gh}_{\mathrm{o}}}}
$$


where $\alpha=C_{s}^{2} \Delta^{2}$ in which $C_{s}=0.2$ is assumed here to be a constant and $\Delta$ is the grid size. The nondimensional vertical eddy viscosity is given by

$$
v_{\mathrm{t}}^{\mathrm{v}}=\frac{v_{\mathrm{t}}^{\mathrm{v}^{*}}}{\beta \mu \mathrm{h}_{\mathrm{o}} \sqrt{\mathrm{gh}_{\mathrm{o}}}}
$$

where the $\beta$ is required to be $\beta=c_{\mathrm{h}} \sqrt{c_{\mathrm{f}}} / \mu$ and $\mathrm{O}(\beta)<<1.0$. With these variables and parameters, the filtered dimensionless form of the continuity equation and the Navier-Stokes equations in the horizontal direction can be derived.

As a next step, by applying the depth-averaging operator, 'tilde', to the filtered dimensionless form of the continuity equation and Navier-Stokes equations, a 2D horizontal equation set can be derived. By the way, during the derivation of a H2D model, the dispersive stresses, or momentum fluxes due to the interaction of velocity fluctuations, are usually ignored following the assumption of a constant vertical velocity profile. Even in the Boussinesq-type model of Kim et al. (2009) that assumes a depthvarying velocity $U_{B}(z)$ as shown in Figure 1 , the fluctuating component $u_{i}^{\prime}$ is ignored. This term, and the associated mixing, can be important for the prediction of kinetic energy transport in environmental flows.

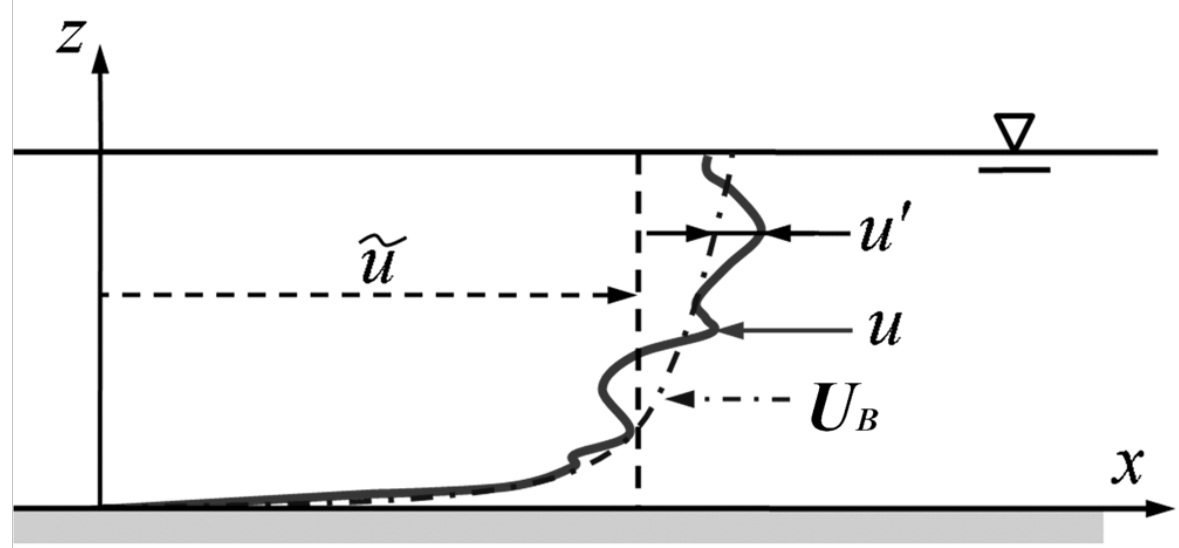

Figure 1. Definitions of velocity notations. $\tilde{u}$ : depth averaged velocity; $u$ : total velocity including fluctuating velocity component; $u^{\prime}$ : spatially fluctuating velocity component; $U_{B}$ : velocity profile by Kim et al. (2009).

To approximate the effects created by the $\mathrm{u}_{\mathrm{i}}$ term, Hinterberger et al. $(2004,2007)$ proposed a stochastic BSM for the shallow water equations. Here, we will incorporate this approach into the Boussinesq-type equations of Kim et al. (2009). First, by integrating the filtered Navier-Stokes equations over the depth, the following equation is obtained:

$$
\begin{aligned}
\frac{\partial H \widetilde{\mathrm{u}}_{1}}{\partial \mathrm{t}} & +\frac{\partial \mathrm{H} \widetilde{\mathrm{u}_{1}} \widetilde{\mathrm{u}}_{j}}{\partial \mathrm{x}_{\mathrm{j}}}+\mathrm{H} \frac{\widetilde{\partial \overline{\mathrm{p}}}}{\partial \mathrm{x}_{\mathrm{i}}}= \\
& \alpha \mu \frac{\partial}{\partial \mathrm{x}_{\mathrm{j}}}\left(2 \mathrm{H} v_{\mathrm{t}}^{\mathrm{h}} \widetilde{\widetilde{\mathrm{S}_{\mathrm{j}}}}\right)-\beta \mu^{2} 2 \frac{\partial}{\partial \mathrm{x}_{\mathrm{i}}}\left(v_{\mathrm{t}}^{\mathrm{v}} \frac{\partial \mathrm{u}_{\mathrm{j}}}{\partial \mathrm{x}_{\mathrm{j}}}\right)-\beta \mu^{2} \tau_{\mathrm{i}}^{\mathrm{b}}-\gamma^{2} \frac{\partial \mathrm{HD}_{\mathrm{ij}}(\overline{\mathrm{u}})}{\partial \mathrm{x}_{\mathrm{j}}}
\end{aligned}
$$

where the depth-averaged velocity is given by

$$
\begin{aligned}
\tilde{u}_{i} & =\frac{1}{H} \int_{H}\left(U_{i}+\mu^{2} u_{i}^{\phi}+\beta \mu u_{i}^{r}+\gamma u_{i}^{\prime}\right) d z+O\left(\mu^{4}, \beta^{2} \mu^{2}\right) \\
& =\frac{1}{H} \int_{H}\left(U_{B i}+\gamma u_{i}^{\prime}\right) d z+O\left(\mu^{4}, \beta^{2} \mu^{2}\right)
\end{aligned}
$$


in which $U_{i}$ is the horizontal velocity at an arbitrary water depth $z_{\alpha}$ and $U_{B i}=U_{i}+\mu^{2} u_{i}^{\phi}+\beta \mu u_{i}^{r}$ and $\mathrm{u}_{\mathrm{i}}^{\phi}$ and $\mathrm{u}_{\mathrm{i}}^{\phi}$ are the second-order rotational and irrotational velocity corrections, respectively (Kim et al., 2009). The newly introduced $\gamma$ is a scale parameter and its magnitude will be assessed later. From the depth-averaging process, equation (6), the dispersive stress $\mathrm{D}_{\mathrm{ij}}(\mathrm{u})$ is given by

$$
\begin{aligned}
D_{i j}(\bar{u}) & =\frac{1}{H} \int_{H}\left(\bar{u}_{i}-\tilde{u}_{i}\right)\left(\overline{u_{j}}-\tilde{u_{j}}\right) d z \\
& =\mu^{2} \gamma\left(\overline{\overline{u_{1}^{\phi}}} \overline{\overline{u_{j}^{\prime}}}+\overline{\overline{u_{1}^{\prime}} \overline{u_{j}^{\phi}}}\right)+\beta \mu \gamma\left(\widetilde{\overline{u_{1}^{r}} \overline{u_{j}^{\prime}}}+\widetilde{\overline{u_{1}^{\prime}} \overline{u_{j}^{r}}}\right)+\gamma^{2} \widetilde{\overline{u_{1}^{\prime}}} \overline{u_{j}^{\prime}}
\end{aligned}
$$

As described in Kim et al. (2009), the bottom friction term can be included through a consistent derivation from the viscous primitive equations; By assuming that the shear stress varies linearly from zero at the water surface to $\tau_{\mathrm{b}}$ at the bottom (Rodi, 1980). Finally, the bottom friction term has the scale of $\beta \mu^{2}$ based on the parameters proposed in this paper. Following this analysis and considering that the typical magnitude of the shear stress is similar to the Reynolds stress, we can deduce $\gamma^{2}=$ $\mathrm{O}\left(\beta \mu^{2}\right)$. Consequently, in the dispersive stress terms in the last line of equation (8), the first and the second terms are smaller than the third (last) term. Thus, only the last term is sustained through the derivation, while the first two terms are lumped within the truncation error of the model, and discarded. Equation (6) then becomes:

$$
\begin{aligned}
\frac{\partial H \widetilde{\mathrm{u}_{1}}}{\partial \mathrm{t}} & +\frac{\partial H \widetilde{\mathrm{u}_{1}} \widetilde{\mathrm{u}_{\mathrm{j}}}}{\partial \mathrm{x}_{\mathrm{j}}}+\mathrm{H} \frac{\widetilde{\partial \overline{\mathrm{p}}}}{\partial \mathrm{x}_{\mathrm{i}}} \\
& =\alpha \mu \frac{\partial}{\partial \mathrm{x}_{\mathrm{j}}}\left(2 \mathrm{H} v_{\mathrm{t}}^{\mathrm{h}} \widetilde{\mathrm{S}_{\mathrm{lj}}}\right)-\beta \mu^{2} 2 \frac{\partial}{\partial \mathrm{x}_{\mathrm{i}}}\left(v_{\mathrm{t}}^{\mathrm{v}} \frac{\partial \mathrm{u}_{\mathrm{j}}}{\partial \mathrm{x}_{\mathrm{j}}}\right)-\beta \mu^{2} \tau_{\mathrm{i}}^{\mathrm{b}}+\gamma^{2} \frac{\partial}{\partial \mathrm{x}_{\mathrm{j}}}\left(\mathrm{H} \widetilde{\overline{\mathrm{u}_{1}^{\prime}}} \widetilde{\mathrm{u}_{\mathrm{j}}^{\prime}}\right) \\
& +O\left(\mu^{4}, \alpha \mu^{3}, \beta \mu^{4}, \mu^{2} \gamma, \beta \mu \gamma\right)
\end{aligned}
$$

\section{Depth-Integrated Eddy Simulation Model}

In this section, the final form of the model equations will be presented, with the employed turbulence closures, in dimensional form. For convenience of expression, all the dimensional variables are given without the superscript ' $*$ ' after dimensionality is recovered. Also, the overbar for the notation of the filtering is not expressed from here. Including the dispersive stress terms above, the depth-integrated equations in conservative form with subgrid scale turbulence closure are given by

$$
\begin{aligned}
& \frac{\partial \zeta}{\partial \mathrm{t}}+\frac{\partial \mathrm{HU}_{\mathrm{i}}}{\partial \mathrm{x}_{\mathrm{i}}}+\mathrm{M}+\mathrm{M}^{v}=0 \\
& \frac{\partial \mathrm{HU}_{\mathrm{i}}}{\partial \mathrm{t}}+\frac{\partial \mathrm{HU}_{\mathrm{i}} \mathrm{U}_{\mathrm{j}}}{\partial \mathrm{x}_{\mathrm{j}}}+\mathrm{gH} \frac{\partial \zeta}{\partial \mathrm{x}_{\mathrm{i}}}+\mathrm{H}\left(\mathrm{D}_{\mathrm{i}}+\bar{\xi}_{\mathrm{I}}+\mathrm{D}_{\mathrm{i}}^{v}+\overline{\xi_{\mathrm{l}}^{v}}\right)+\mathrm{U}_{\mathrm{i}}\left(\mathrm{M}+\mathrm{M}^{v}\right) \\
& \quad-\mathrm{H} \frac{\partial}{\partial \mathrm{x}_{\mathrm{j}}}\left(2 v_{\mathrm{t}}^{\mathrm{h}} \mathrm{S}_{\mathrm{ij}}\right)+2 \mathrm{H} \frac{\partial}{\partial \mathrm{x}_{\mathrm{i}}}\left(v_{\mathrm{t}}^{\mathrm{v}} \frac{\partial \mathrm{U}_{\mathrm{j}}}{\partial \mathrm{x}_{\mathrm{j}}}\right)+\frac{\tau_{\mathrm{i}}^{\mathrm{b}}}{\rho}-\mathrm{HF}_{\mathrm{i}}=0
\end{aligned}
$$

in which $i, j=(1,2), M$ and $\mathbf{M}^{v}$ are the second-order dispersion and vorticity correction terms of the continuity equation, respectively. In the momentum equation, $\mathrm{D}_{\mathrm{i}}$ and $\mathrm{D}_{\mathrm{i}}{ }^{v}$ are the frequency dispersion corrections due to free surface waves and bottom-generated turbulence respectively. $\bar{\xi}_{1}$ and $\xi_{i}^{v}$ are the rotational corrections due to free surface waves and turbulence respectively. More details about these higher-order terms and other terms can be found in Kim et al. (2009).

The $\mathrm{HF}_{\mathrm{i}}$ term representing the dispersive stresses in equation (11) is implemented with a stochastic BSM proposed by Hinterberger et al. (2004, 2007).

$$
F_{i}=C_{B} \frac{\sqrt{\widetilde{\mathrm{u}}^{2}+\widetilde{\mathrm{v}}^{2}}}{\mathrm{H}} \sqrt{\frac{v \sqrt{\mathrm{cf}}}{\Delta \mathrm{t}}} \mathrm{r}_{\mathrm{i}}
$$

\section{DEPTH-INTEGRATED TRANSPORT MODEL}

Here, Taylor's analysis is followed, in essence, to derive a depth-integrated transport equation for a scalar property. The same long wave scaling and dimensional analysis as used previously for the 
hydrodynamic model is applied. With the turbulent diffusion expressed as $v_{\mathrm{t}}^{*} / \sigma_{\mathrm{t}}$ and the magnitude of turbulent Schmidt number is assumed to be 1.0, the turbulent diffusion scaling can be given by

$$
\left(D_{x}, D_{y}\right)=\frac{\left(D_{x}^{*}, D_{x}^{*}\right)}{\alpha h_{o} \sqrt{g_{o}}}, \quad D_{z}=\frac{D_{z}^{*}}{\beta \mu h_{o} \sqrt{g_{o}}}
$$

where $D_{x}$ and $D_{y}$ are the nondimensional horizontal turbulent diffusion terms and $D_{z}$ is the nondimensional vertical turbulent diffusion. Applying the perturbation approach with the scaling in (3) and (13) and by depth-integration, the 3D advection-diffusion equation for some scalar concentration C can be transformed to

$$
\frac{\partial H \widetilde{\mathrm{C}}}{\partial \mathrm{t}}+\frac{\partial H \widetilde{\mathrm{u}} \widetilde{\mathrm{C}}}{\partial \mathrm{x}_{\mathrm{i}}}=\frac{\partial}{\partial \mathrm{x}_{\mathrm{i}}}\left(\mathrm{HD}_{\mathrm{Lij}} \frac{\partial \widetilde{\mathrm{C}}}{\partial \mathrm{x}_{\mathrm{j}}}\right)+\frac{\partial}{\partial \mathrm{x}_{\mathrm{i}}}\left(\mathrm{HD}_{\mathrm{Lij}} \frac{\partial \widetilde{\mathrm{C}}}{\partial \mathrm{x}_{\mathrm{i}}}\right)
$$

where $D_{x i}$ is horizontal turbulent coefficient and given by $D_{x i}=v_{t}^{*} / \sigma_{t} \cdot D_{L i j}$ is the dispersion coefficient and given by

$$
D_{\mathrm{Lij}}=-\frac{1}{\mathrm{H}} \int_{\mathrm{H}} \mathrm{u}_{\mathrm{i}}^{\prime \prime} \int_{-\mathrm{h}}^{\mathrm{z}} \frac{1}{\mathrm{D}_{\mathrm{z}}} \int_{-\mathrm{h}}^{\mathrm{z}} \mathrm{u}_{\mathrm{j}}^{\prime \prime} \mathrm{dzdzdz}
$$

in which $\mathrm{u}$ "is the deviation of the velocity and the dispersion coefficient can be expressed explicitly via equation (7) using the analytical vertical velocity profile of the Kim et al. (2009) model. After some algebra,

$$
\begin{aligned}
\mathrm{D}_{\mathrm{Lij}}= & -\frac{\sigma_{\mathrm{t}}}{\mathrm{C}_{\mathrm{h}} \mathrm{H}^{2} \mathrm{u}_{\mathrm{\tau}}}\left[\frac{1}{336}\left(\phi_{\mathrm{i}}+\frac{\partial \mathrm{S}}{\partial \mathrm{x}_{\mathrm{i}}}\right)\left(\psi_{\mathrm{j}}+\frac{\partial \mathrm{S}}{\partial \mathrm{x}_{\mathrm{j}}}\right)\left(\zeta^{7}+\mathrm{h}^{7}\right)\right. \\
& +\frac{1}{48}\left(\phi_{\mathrm{i}}+\frac{\partial \mathrm{S}}{\partial \mathrm{x}_{\mathrm{i}}}\right)\left(\frac{\partial \mathrm{T}}{\partial \mathrm{x}_{\mathrm{j}}}-\zeta \psi_{\mathrm{j}}\right)\left(\zeta^{6}-\mathrm{h}^{6}\right) \\
+ & \frac{1}{30}\left(\frac{\partial \mathrm{T}}{\partial \mathrm{x}_{\mathrm{i}}}-\zeta \psi_{\mathrm{i}}\right)\left(\frac{\partial \mathrm{T}}{\partial \mathrm{x}_{\mathrm{j}}}-\zeta \psi_{\mathrm{j}}\right)\left(\zeta^{5}+\mathrm{h}^{5}\right) \\
& -\frac{7}{120}\left(\frac{\partial \mathrm{S}}{\partial \mathrm{x}_{\mathrm{i}}}+\psi_{\mathrm{i}}\right)\left(\mathrm{C}_{4 \mathrm{j}}-\mathrm{C}_{2} \psi_{\mathrm{j}}\right)\left(\zeta^{5}+\mathrm{h}^{5}\right) \\
& -\frac{1}{6}\left(\frac{\partial \mathrm{T}}{\partial \mathrm{x}_{\mathrm{i}}}-\zeta \psi_{\mathrm{i}}\right)\left(\mathrm{C}_{4 \mathrm{j}}-\mathrm{C}_{2} \psi_{\mathrm{j}}\right)\left(\zeta^{4}-\mathrm{h}^{4}\right) \\
& -\frac{1}{8}\left(\frac{\partial \mathrm{S}}{\partial \mathrm{x}_{\mathrm{i}}}+\psi_{\mathrm{i}}\right)\left(\mathrm{C}_{5 \mathrm{j}}-\mathrm{C}_{3} \psi_{\mathrm{j}}\right)\left(\zeta^{4}-\mathrm{h}^{4}\right) \\
& +\frac{1}{6}\left(\mathrm{C}_{4 \mathrm{i}}-\mathrm{C}_{2} \psi_{\mathrm{i}}\right)\left(\mathrm{C}_{4 \mathrm{j}}-\mathrm{C}_{2} \psi_{\mathrm{j}}\right)\left(\zeta^{3}+\mathrm{h}^{3}\right) \\
& -\frac{1}{3}\left(\frac{\partial \mathrm{T}}{\partial \mathrm{x}_{\mathrm{i}}}-\zeta \psi_{\mathrm{i}}\right)\left(\mathrm{C}_{5 \mathrm{j}}-\mathrm{C}_{3} \psi_{\mathrm{j}}\right)\left(\zeta^{3}+\mathrm{h}^{3}\right) \\
& -\frac{1}{6}\left(\frac{\partial \mathrm{S}}{\partial \mathrm{x}_{\mathrm{i}}}+\psi_{\mathrm{i}}\right) \mathrm{C}_{1 \mathrm{j}}\left(\zeta^{3}+\mathrm{h}^{3}\right) \\
& +\frac{1}{2}\left(\mathrm{C}_{5 \mathrm{i}}-\mathrm{C}_{3} \psi_{\mathrm{i}}\right)\left(\mathrm{C}_{4 \mathrm{j}}-\mathrm{C}_{2} \psi_{\mathrm{j}}\right)\left(\zeta^{2}-\mathrm{h}^{2}\right) \\
& -\frac{1}{2}\left(\frac{\partial \mathrm{T}}{\partial \mathrm{x}_{\mathrm{i}}}-\zeta \psi_{\mathrm{i}}\right) \mathrm{C}_{1 \mathrm{j}}\left(\zeta^{2}-\mathrm{h}^{2}\right) \\
& +\mathrm{C}_{1 \mathrm{j}}\left(\mathrm{C}_{4 \mathrm{j}}-\mathrm{C}_{2} \psi_{\mathrm{j}}\right)(\zeta+\mathrm{h})
\end{aligned}
$$

Where

$$
\begin{aligned}
\mathrm{C}_{1 \mathrm{i}} & =\mathrm{h}\left(\mathrm{C}_{5 \mathrm{i}}-\mathrm{C}_{3} \psi_{\mathrm{i}}\right)-\frac{1}{2} \mathrm{~h}^{2}\left(\mathrm{C}_{4 \mathrm{i}}-\mathrm{C}_{2} \psi_{\mathrm{i}}\right)+\frac{1}{6} \mathrm{~h}^{3}\left(\zeta \psi_{\mathrm{i}}-\frac{\partial \mathrm{T}}{\partial \mathrm{x}_{\mathrm{i}}}\right)+\frac{1}{24} \mathrm{~h}^{4}\left(\frac{\partial \mathrm{S}}{\partial \mathrm{x}_{\mathrm{i}}}+\psi_{\mathrm{i}}\right) \\
\mathrm{C}_{2} & =\frac{1}{6}\left(2 \zeta^{2}-2 \zeta \mathrm{h}-\mathrm{h}^{2}\right) \\
\mathrm{C}_{3} & =\frac{1}{6} \zeta \mathrm{h}(\mathrm{h}+2 \zeta)
\end{aligned}
$$




$$
\begin{aligned}
& C_{4 i}=\frac{1}{6}\left(\zeta^{2}-\zeta h+h^{2}\right) \frac{\partial S}{\partial x_{i}}+\frac{1}{2}(\zeta-h) \frac{\partial T}{\partial x_{i}} \\
& C_{5 i}=-\frac{1}{6} h^{3} \frac{\partial S}{\partial x_{i}}+\frac{1}{2} h^{2} \frac{\partial T}{\partial x_{i}}+C_{4 i} h
\end{aligned}
$$

where the definitions of the each terms can be found in Kim et al. (2009). The tedious expression can be verified through a look at the case of uniform flow. With uniform flow, $\kappa / 6=0.0667$ from the standard von Karman value, and $\sigma_{t}=0.8302$, equation (16) yields the dispersion coefficient $5.93 \mathrm{Hu}_{\tau}$. Thus, for application of the transport model in future sections of this paper, $\sigma_{t}=0.8302$ is used.

\section{NUMERICAL METHOD}

The fourth-order accurate MUSCL FVM (Yamamoto and Daiguji, 1993) with HLL Riemann solver (Toro, 1997) is used for the advection terms in equation (14). For the diffusion terms, fourthorder finite volume discretization equations are used. The time integration utilizes the third-order Adams-Bashforth predictor and the fourth-order Adams-Moulton corrector scheme. Details of the numerical method for flow model are well described in Kim and Lynett (in press, b). For the transport equation, detail numerical methods are described in Kim and Lynett (in press, a). In the reference, several typical tests to demonstrate the accuracy of the transport model are provided.

\section{TURBULENT FLOW AND TRANSPORT SIMULATIONS}

\section{Mixing layer Simulations}

To investigate the mixing by internal transverse shear instability, flow in a plane mixing layer experimented by Babarutsi and Chu (1998) is simulated with the depth-integrated model with BSM model. The dimension of the channel is $0.61 \mathrm{~m}$ wide, $7 \mathrm{~m}$ long, and the water depth $\mathrm{h}=0.0296 \mathrm{~m}$. The inflow section is divided by a plate as shown in Figure 2. At the upstream boundary, the velocity on one side of the plate, in the lower half of the channel, is $\mathrm{u}_{1}=0.111 \mathrm{~m} / \mathrm{s}$ and the velocity on the other side is $\mathrm{u}_{2}=0.264 \mathrm{~m} / \mathrm{s}$. For the numerical simulation, the grid size $=0.2 \mathrm{~h}$ and $\mathrm{C}_{\mathrm{B}}=100$ are used.

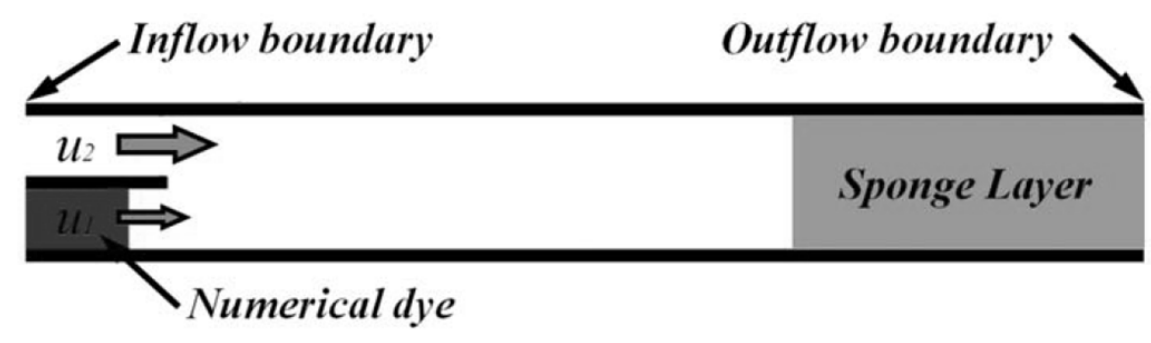

Figure 2. Schematic diagram of Mixing Layer

Figure 3 shows the $<\mathrm{u}>$ (time mean velocity) and the $\mathrm{u}_{\text {rms }}$ (root mean square value of the velocity fluctuation in the streamwise direction) by the depth-integrated flow model with BSM and the measurement. The discrepancy around the downstream mainly resulted from the different downstream boundary conditions between the experiment and the numerical simulation, and from the freeslip condition at the side walls in the numerical model. However, the computed $<\mathrm{u}\rangle$, the slope of the $<\mathrm{u}>$ profile into the transverse direction and the spreading rate of the mixing layer agree well with the experimental data in overall sense. These good agreements are observed again in the comparison of the $\mathrm{u}_{\text {rms }}^{\prime}$, so reasonable prediction of the energy transport and scalar mixing by turbulent flow are expected.

However, without the BSM, that is only with the hydrodynamic model, the spreading rate and the slope of the $<\mathrm{u}>$ do not agree with the experimental data as shown in the Figure 4 . Especially, the value of the $\mathrm{u}_{\text {rms }}^{\prime}$ is too small and even the tendency is absolutely different: The computational results are continuously getting bigger as flows toward the downstream unlike the experimental data. The main reason of the overall discrepancies resulted from that the strength of the horizontal shear of the numerical model without BSM is not strong enough to destabilize in the mixing layer. Therefore the 3D turbulence effects in the equation (9) should be included in the case of destabilization by internal transverse shear. 

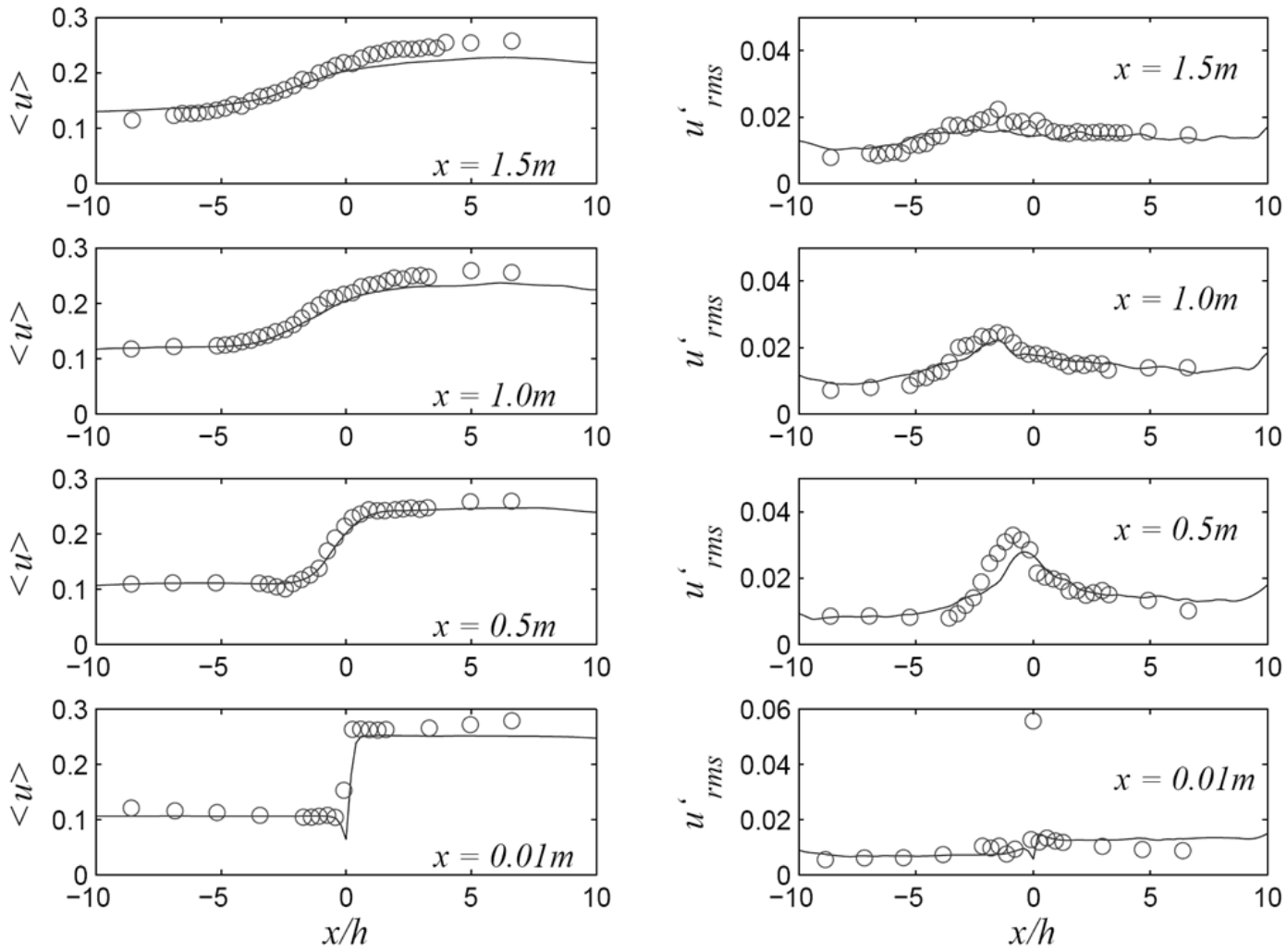

Figure 3. Comparisons of the depth-integrated flow model with BSM results and experimental data. Left: time mean velocity $(\mathrm{m} / \mathrm{s})$. Right: root mean square velocity $(\mathrm{m} / \mathrm{s})$. Circle: experimental data (by Babarutsi and Chu ,1998), line: numerical results.
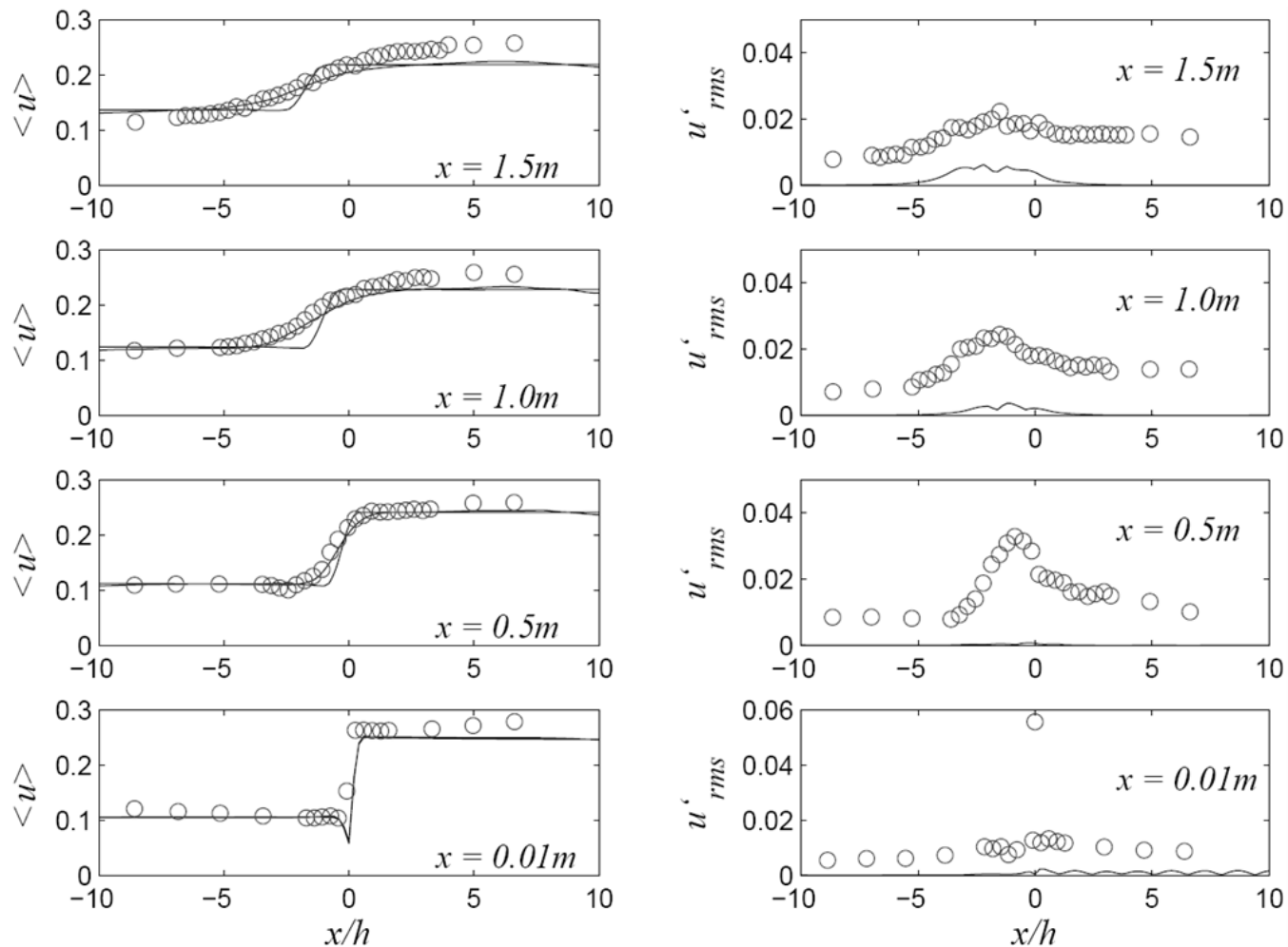

Figure 4. Comparisons of the numerical results without BSM and experimental data. Left: time mean velocity (m/s). Right: root mean square velocity (m/s). Circle: experimental data (by Babarutsi and Chu, 1998), line: numerical results. 


\section{Comparison to Taylor's Theorem}

Rummel et al. (2005) presented the results of an experimental study to determine the magnitude of mixing coefficient for a passive tracer plume in shallow open channel flow. Also they presented two analytical solutions for the near field and far field mixing based on the Taylor's theorem (1921). These results are compared with the numerical simulation results quantitatively in this section. From the solutions, we can see that the $\sigma_{\mathrm{y}} / \mathrm{h}$ is a function of the transverse turbulent intensity and proportional to $(\mathrm{x} / \mathrm{h})$ in the near field and proportional to $(\mathrm{x} / \mathrm{h})^{0.5}$ in the far field. Hence, numerical results should be related with the turbulent intensity and should show the similar proportionality with the analytical solutions in the near and far fields, respectively. In the laboratory experiment, the flow conditions resulted in the turbulent intensity $i_{y}=0.06$. In the numerical simulation, the $C_{B}=70$ resulted in the $\mathrm{i}_{\mathrm{y}}=0.06$ and randomly distributed scalar distribution. In the experiment done by Rummel et al. (2005), the channel length was $13.5 \mathrm{~m}$ and the width was $5.5 \mathrm{~m}$. The water depth was $\mathrm{h}=0.025 \mathrm{~m}$ and the velocity was $\mathrm{U}=0.16 \mathrm{~m} / \mathrm{s}$, resulting in the $\mathrm{Re}=4000$. The bed friction coefficient was given by $\mathrm{f}=0.029$ from the experiment. The dye was injected through a $0.001 \mathrm{~m}$ diameter tube into the streamwise direction constantly at the middepth.

The Figure 5 shows the characteristic plume half widths by the experiment and the numerical simulation. In far field, the slope (qualitative characteristic) and the width (quantitative characteristic) agree well each other. In near field, the slopes of the analytic solution and the numerical and experimental data show a little difference. It resulted from the limitation of 2D horizontal model. That is, in near field, the flow in numerical model is still 2D motion in overall sense, but contains highly 3D small scale turbulence in real spaces which cannot be resolved by a 2D horizontal model. Looking back to the derivation of the depth-integrated transport equation, during the derivation of depth-integrated transport equation several assumptions which are not valid in near field were applied (Kim and Lynett, in press, a). Therefore, a different dispersion model specialized for the near field or a 3D model is required for more accurate near field mixing simulations.

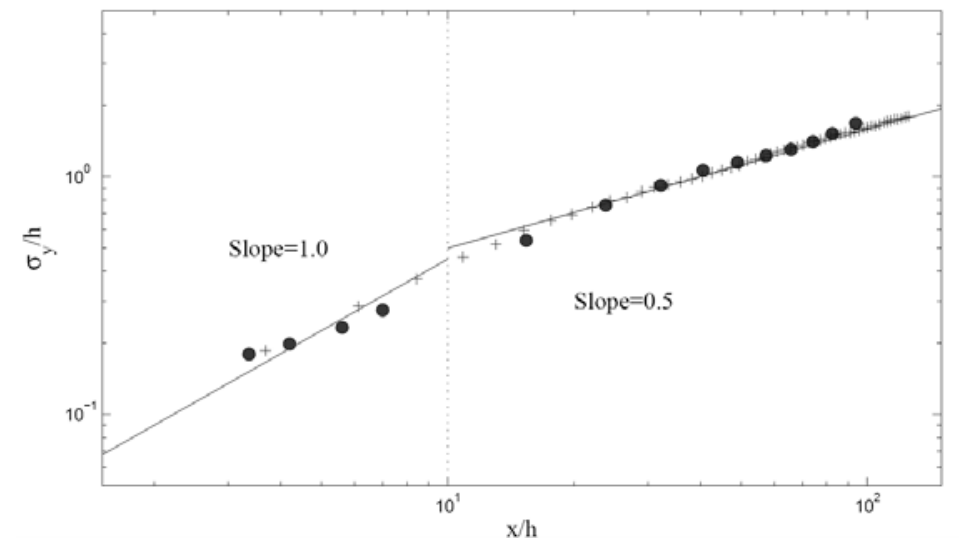

Figure 5. Normalized standard deviation $\sigma y / h$ of the transverse concentration profiles. Line: analytic (by Rummel et al, 2005), circle: computed results, cross: measured data (by Rummel et al, 2005).

\section{Mixing by Topographical Forcing}

It is greatly important to investigate the effects of topography like islands to flows, because their effects that can cause instability and coherent structures are very strong (Jirka, 2002). Thus in this section, the mixing by the $2 \mathrm{D}$ coherent structures generated by the typical islands is investigated.

For the investigation, the flows studied by a laboratory experiment and numerical model were selected. The laboratory experiment was conducted by Lloyd and Stansby (1997). The experimental setup was: The $0.049 \mathrm{~m}$ high island with 8 degree side slope was installed at the $5.0 \mathrm{~m}$ downstream from the inlet. The channel length was $9.75 \mathrm{~m}$ and the width was $1.52 \mathrm{~m}$. The outer radius and the inner radius of the island were $0.375 \mathrm{~m}$ and $0.025 \mathrm{~m}$, respectively. A steady streamwise flow with velocity $0.115 \mathrm{~m} / \mathrm{s}$ was released at the upstream boundary. Two different cases were tested. The water depth was $0.054 \mathrm{~m}$. Thus the Reynolds numbers are $\mathrm{Re}=6210$. For the numerical simulation, the grid size is $0.01 \mathrm{~m}$, the 
$C_{B}=70$ and the $C r=0.5$ were used. For the scalar transport simulations, the numerical dye is injected at the $0.5 \mathrm{~m}$ upstream of the island.

For the submerged island case, the water depth was so shallow at the apex of the island that the separation was observed at the downstream lip of the horizontal apex and across the upper shoulder (Lloyd and Stansby, 1997). Thus, the 2D coherent structure can be generated by the topographical forcing. The Figure 6 shows the computed results by the depth-integrated flow model with BSM model and the computed concentration looks similar to the experimental data in Lloyd and Stansby (1997). The 2D coherent structures were generated very reasonably without the BSM as shown in the Figure 7. However, it will be very hard to decide whether the effects of the 3D turbulence is important or not in real applications, because the topography is so arbitrary. Hence it is recommended include the BSM for an important simulation. In conclusion, the proposed depth-integrated flow model with BSM model is expected to be able to predict the mixing by topographical change.
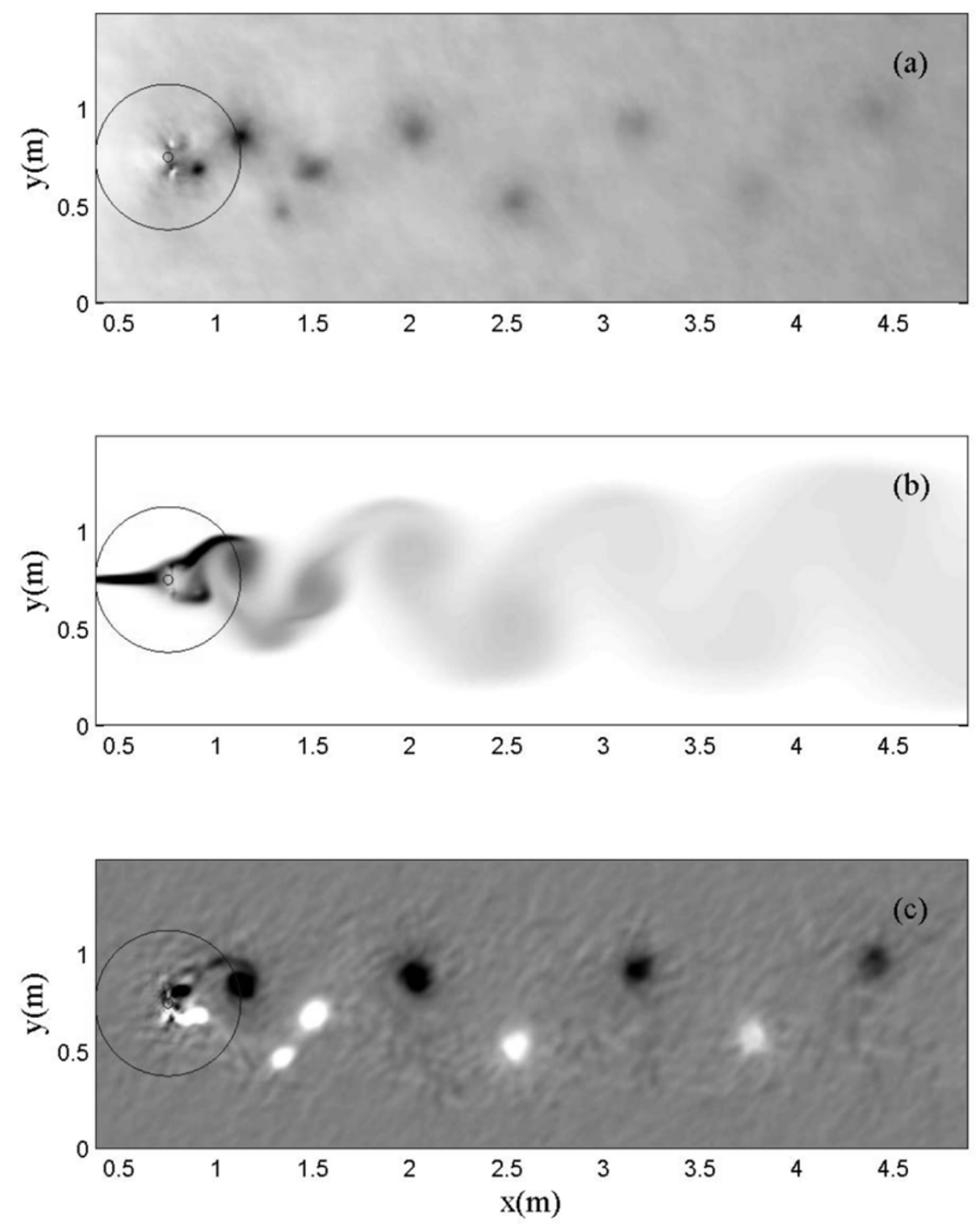

Figure 6. Computed results for submerged case by DEPTH-INTEGRATED FLOW MODEL. (a) water surface elevation, (b) scalar concentration, (c) vertical vorticity.. 

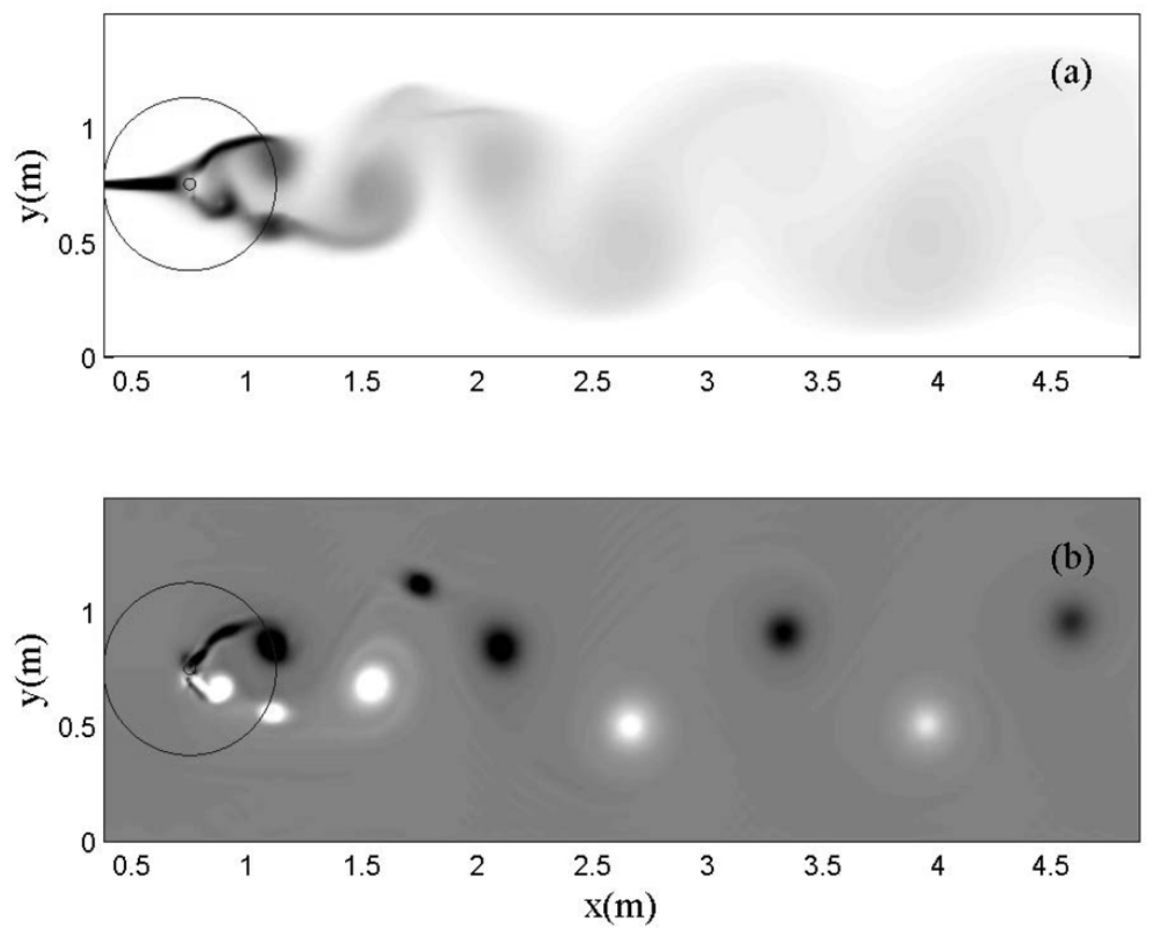

Figure 7. Computed results without BSM for submerged island case. (a) scalar concentration, (b) vertical vorticity.

\section{SUMMARY}

For the turbulent transport by long waves and currents, the 3D turbulent effects were incorporated by a perturbation approach into the fully nonlinear Boussinesq equations which are for weakly dispersive and rotational flow.

A depth-integrated scalar transport equations were derived by the same perturbation approach based on the long wave scaling for consistency. The dispersion coefficient was derived based on the vertical velocity profile of the Boussinesq-type equations. The proposed equations were solved by a fourthorder accurate FVM. Several typical tests for the verifications of the scalar transport solver showed very good agreements with analytical solutions. Especially, very little error by numerical dispersion, dissipation and diffusion were detected.

From the mixing layer simulation with the stochastic BSM, the importance of the 3D turbulence effects to the turbulent transport was apparently proved. The comparisons with the Taylor's theorem showed that the proposed depth-integrated transport model coupled with the DISGS model has the consistency with the analytic solutions and the experimental data in far field. From the comparison in near field, the inherent limitation of the $2 \mathrm{D}$ horizontal model was recognized. In the simulations of the mixing by the bottom topography, the effects of the 3D turbulence effects were less important than the other cases.

\section{REFERENCES}

Alvelius, K. (1999) Random Forcing of Three-Dimensional Homogeneous Turbulence, Physics of Fluids, vol.11, pp.1880-1889.

Babarutsi, S. and Chu, V. H. (1998) Modeling Transverse Mixing Layer in Shallow Open-Channel Flows. Journal of Hydraulic Engineering, ASCE, vol.124, no.7, pp.718-727.

Chen, D. and Jirka, G.H. (1995) Experimental Study of Plane Turbulent Wakes in a Shallow Water Layer, Fluid Dynamics Research, vol.16, pp.11-41. 
Elder, J.W., (1959) The dispersion of marked fluid in turbulent shear flow. Journal of Fluid Mechanics, vol.5, pp.546-560.

Fischer, H. B., List, E. J., Koh, R. C. Y., Imberger, J., and Brooks, N. H. (1979) Mixing in inland and coastal waters, Academic press, New York.

Hinterberger, C., Frohlich, J. and Rodi, W. (2004) Three-Dimensional and Depth-Averaged LargeEddy-Simulation of Shallow Water Flows, Shallow flows, pp.567-574.

Hinterberger, C., Frohlich, J. and Rodi, W. (2007) 3D and Depth-Averaged Large-Eddy Simulations of Some Shallow Water Flows, Journal of Hydraulic Engineering, ASCE, vol.133, no.8, pp.857-872.

Jirka (2001) Large Scale Flow Structures and Mixing Processes in Shallow Flows, Journal of Hydraulic Research. vol.39, no.6, pp.567-573.

Kim, D.H., Lynett, P., and Socolofsky, S. (2009) A Depth-Integrated Model for Weakly Dispersive, Turbulent, and Rotational Fluid Flows, Ocean Modelling, vol.27, pp.198-214.

Kim, D.H. and Lynett, P. (in press, a) Turbulent Mixing and Scalar Transport in Passive Shallow Flows. Physics of Fluids.

Kim, D.H. and Lynett, P. (in press, b) Dispersive and Nonhydrostatic Pressure Effects at the Front of Surge, Journal of Hydraulic Engineering.

Kuipers, J.and Vreugdenhill, C.B. (1973) Calculations of 2D horizontal flow, Delft Hydraulics. Lab. Report, S163, part 1.

Lloyd, P.M. and Stansby, P.K. (1997) Shallow Water Flow around Model Conical Island of Small Slope. II: Submerged, Journal of Hydraulic Engineering, ASCE, vol.123, no.12, pp.1068-1077.

Mingham, C.G. and Causon, D.M. (2008) A simple high-resolution advection scheme, International Journal for Numerical Methods in Fluids, vol.56, pp.469-484.

Nadaoka, K., and Yagi, H. (1998) Shallow-Water Turbulence Modeling and Horizontal Large-Eddy Computation of River Flow, Journal of Hydraulic Engineering, vol.124, no.5, pp.493-500.

Nwogu, O. (1993) Alternative Form of Boussinesq Equations for Nearshore Wave Propagation, Journal of Waterway, port, Coastal, and Ocean Engineering, vol.119, no.6, pp.618-638.

Rodi, W. (1980) Turbulence models and their application in hydraulics - a state of the art review, IAHR.

Rummel, A.C., Socolofsky, S.A., Carmer, C.F.V., and Jirka, G.H. (2005) Enhanced diffusion from a continuous point source in shallow free-surface flow with grid turbulence, Physics of Fluids, vol.17.

Taylor, G. I. (1921) Diffusion by continuous movements, Proceedings of the London Mathematical Society, vol.20, pp.196-212.

Taylor, G. I. (1953) Dispersion of soluble matter in a solvent flowing slowly through a tube, Proceedings of the Royal Society of London, Series A, vol.219, pp.186-203.

Toro, E.F. (1997) Riemann Solvers and Numerical Methods for Fluid Dynamics. Springer-Verlag.

Toro, E.F. (2002) Shock-Capturing Methods for Free-Surface Shallow Flows, John Wiley \& Sons, Ltd. 
Yamamoto, S. and Daiguji, H. (1993) Higher-Order-Accurate Upwind Schemes for Solving the Compressible Euler and Navier-Stokes Equations, Computers and Fluids, vol.22, no.2/3, pp.259270. 